\title{
Development of motor body fixture using blackboard framework approch
}

\author{
Mr. A. D. PARSANA \\ M.E.[Machine Design] Student, Department Of Mechanical Engineering, R. K. College Of Engineering And Technology, \\ Rajkot, Gujarat \\ Prof. A. P. SAVALIA \\ Professor, Department Of Mechanical Engineering, R. K. College Of Engineering And Technology, Rajkot, Gujarat
}

\begin{abstract}
:-
An approach based on blackboard frame work techniques is proposed to develop an automated fixturing system that can interpret the semantic information of features of a 3-D object, created in a computer-aided design system, and suggest the possible clamping conditions on the object. This paper reports the preliminary work that investigates the suitability of using a blackboard framework as a problem-solving model to realize such a system. This system is developed for the development of motor stator body fixture for HMC machine. With help of this system designer can easily design and manufacture new fixture for motor stator body.
\end{abstract}

Keywords: - Fixture development, Fixture principle, Black-board framework.

\section{Introduction}

A key concern for manufacturing companies is developing the ability to design and produce a variety of high quality products within short timeframes. As a result of the consumer desire for variety, batch production of products is now more the norm than mass production, which has resulted in the need for manufacturers to develop flexible manufacturing practices to achieve a rapid turnaround in product development [A-D]. Fixtures are used to rapidly, accurately, and securely position workpieces during machining such that all machined parts fall within the design specifications for that part. This accuracy facilitates the inter-changeability of parts that is prevalent in much of modern manufacturing where many different products feature common parts [E-F]. Fixture design is a complicated, experience based process which needs comprehensive qualitative knowledge about a number of design issues including workpiece configuration, manufacturing processes involved, machining environment, etc [C]. Various computer-based systems have been developed in recent years to achieve automation of the fixture design process. These systems use different knowledge sources (KSs), methodologies and system platforms to form a fixture design problem-solving model, and are able to propose feasible fixture configurations only. In a real it is expected that the embedded fixture design system should be able to deal with any generic fixture design process so that a complete automation of the overall manufacturing process is possible [I]. To achieve this, it is required to provide an appropriate fixture design environment which fosters communication between various KS (experts) Involved in the fixture design process [J-K].

\section{Fixture development}

Fig. 1 represents a typical example of a fixture in which the workpiece rests on locators that accurately locate it. Clamps hold the workpiece against the locators during machining thus securing the workpiece's location. The locating units themselves consist of the locator supporting unit and the locator that contacts the workpiece. The clamping units consist of a clamp supporting unit and a clamp that contacts the workpiece and exerts a clamping force to restrain it. Typically the development process by which such fixtures are created has four phases: setup planning, fixture planning, unit design, and verification, as illustrated in Fig. 2. There has been a considerable amount of research conducted with regard to the development of CAFD approaches and subsequent software implementations of those tools. In particular, CAFD research has focused upon setup planning, fixture planning, and verification, in which fixture planning and verification have been closely integrated with respect to minimizing workpiece deformation by controlling the locating and clamping positions.

Recent successes in artificial intelligence (AI), together with increased availability of expert systems development tools, have spurred much interest in the application of AI techniques to develop intelligent fixture design systems. Darvishi and Gill developed a fixture design system with a rule-based knowledge representation database in the OPS5 environment. Their system attempts to utilize the existing expertise in the evolution of a logical automated approach towards the fixture design. 


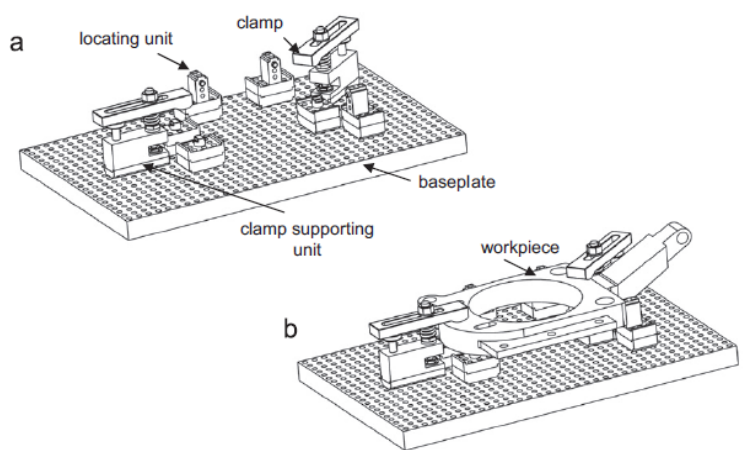

Fig. 1. A typical fixture (a) without and (b) with a workpieces[2].

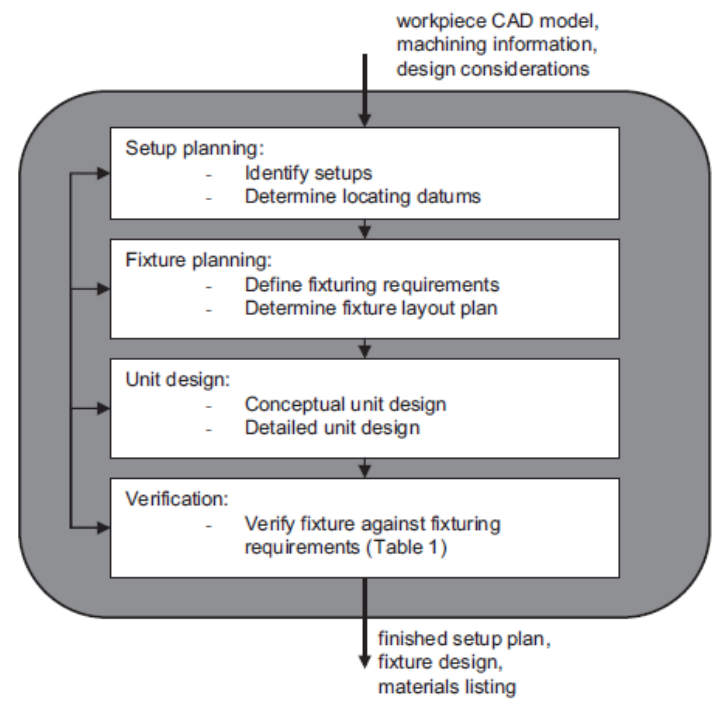

Fig. 2, The fixture design process[1]

\section{Blackboard based design system}

The blackboard system consists of three major components: the KSs, the blackboard data structure and the control structure. Other than the core blackboard module developed in GBB's environment, the system also consults with other supporting modules residing in ICAD's Concept Modeler and in I-DEAS as shown in Fig. 3 in its decision making processes. The different components of the system framework are described in the following subsections.

1. Knowledge sources (KSs)

2. Design heuristics.
3. Fixturing stability.
4. Processing stability
5. Fixturing accessibility.
6. Deformation.

\section{Blackboard data structure}

Referred to usually as the 'blackboard', it provides a hierarchical organization of global state data. It records and organizes the solution elements generated during the problem solving process. The KSs produce changes to the blackboard that lead incrementally to a solution, or a set of acceptable solutions, to the problem. Interaction among the KSs takes place solely through changes on the blackboard.

Fig. 2 illustrates an example of a hierarchical, blackboard data structure created in GBB. There are two basic components in the blackboard structure, spaces e.g., locations and clamps., and units e.g point and event. Spaces divide the whole blackboard data structure into tree-like directories for residing objects called units. The spaces and units in a blackboard structure are like directories and files in a file system. The properties of units are determined by their various attributes, or slots e.g., name, link, path, $x, y$, and $z$ coordinates, etc. During the problem solving process, information resident in slots associated with objects (units) on one level serves as input to a set of KSs, which place new information on the same or other levels in turn. The objects and their properties define the vocabulary of the solution space.

\section{Fixture layout}

Fixture is the component which will locket, clamp a workpiece and retain the position of the workpiece at the time of machining. Fixtures are used to rapidly, accurately, and securely position workpieces during machining such that all machined parts fall within the design specifications for that part. Machining of Motor stator body is a very complicated process due to its wall thickness and geometry. So the development of fixture for motor stator body is needs lots of experience and knowledge about the body. As we are planning a multiple motor series machining changing one or two component in fixture assembly.

We are planning a multiple motor series machining changing one or two component in fixture assembly. 
Table 1. Motor Body Dimensions

\begin{tabular}{|l|c|c|c|c|c|c|c|c|c|c|c|c|}
\hline \multicolumn{1}{|c|}{ Motor Body } & Bore & Tolerance & $\begin{array}{c}\text { Total } \\
\text { length }\end{array}$ & Tolerance & $\begin{array}{c}\text { Spigot } \\
\text { O.D }\end{array}$ & Tolerance & $\begin{array}{c}\text { Spigott } \\
\text { Length }\end{array}$ & Tolerance & I.D. & Tolerance & $\begin{array}{c}\text { I.D. } \\
\text { length }\end{array}$ & Tolerance \\
\hline & & & & & & & & & & & & \\
\hline ABB-315 SM & 520.500 & 0.050 & 640.000 & 0.300 & 536.000 & 0.022 & 4.000 & 0.100 & 521.000 & 0.200 & 100.000 & 1.000 \\
\hline ABB-315 ML & 520.500 & 0.050 & 700.000 & 0.300 & 536.000 & 0.022 & 4.000 & 0.100 & 521.000 & 0.200 & 100.000 & 1.000 \\
\hline ABB-280 & 445.500 & 0.050 & 612.000 & 0.100 & 460.000 & 0.022 & 4.000 & 0.100 & 446.000 & 0.100 & 135.000 & 1.000 \\
\hline Siemense 7015 & 389.950 & 0.050 & 495.000 & 0.300 & 395.000 & 0.036 & 10.000 & 0.100 & 391.000 & 0.300 & 28.000 & 1.000 \\
\hline Siemense 7016 & 434.920 & 0.060 & 555.000 & 0.400 & 440.000 & 0.040 & 10.000 & 0.100 & 436.000 & 0.600 & 28.000 & 1.000 \\
\hline Siemense 7017 & 489.920 & 0.070 & 625.000 & 0.400 & 495.000 & 0.040 & 11.000 & 0.100 & 491.000 & 0.500 & 32.500 & 1.000 \\
\hline CG-225 & 368.350 & 0.050 & 405.000 & 0.100 & 371.060 & 0.060 & 12.000 & 0.100 & & & & \\
\hline CG-250 & 394.090 & 0.040 & 440.000 & 0.100 & 395.280 & 0.050 & 10.000 & 0.100 & & & & \\
\hline CG-280 & 419.150 & 0.050 & 580.000 & 0.100 & 428.620 & 0.050 & 10.000 & 0.100 & & & & \\
\hline BG-315 & 469.800 & 0.050 & 620.000 & 0.100 & 470.530 & 0.060 & 10.000 & 0.100 & & & & \\
\hline BBL-315 & 439.980 & 0.070 & 545.000 & 0.250 & 452.000 & 0.060 & 10.000 & 0.100 & 440.200 & 0.500 & 100.000 & 1.000 \\
\hline
\end{tabular}

*All Dimension are in $\mathrm{mm}$.

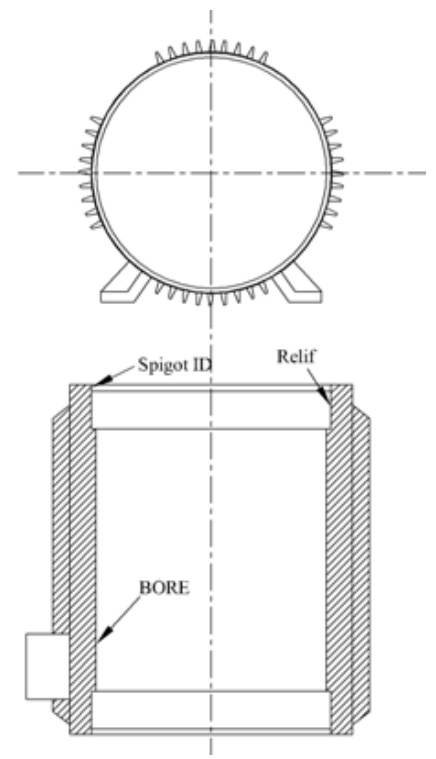

Fig. 3 Motor stator body

Following parameter are useful for fixture design :

- Locating position

- Resting position

- Clamping position

- Machining reference position

- Accuracy of machined parts

- Material of the workpiece

- Weight of workpiece

Main surfaces of the motor stator body are as shown in the fig, which can be used for the locating, clamping and resting during HMC machining process. A steps for fixture development process is as given in the fig. 4 .

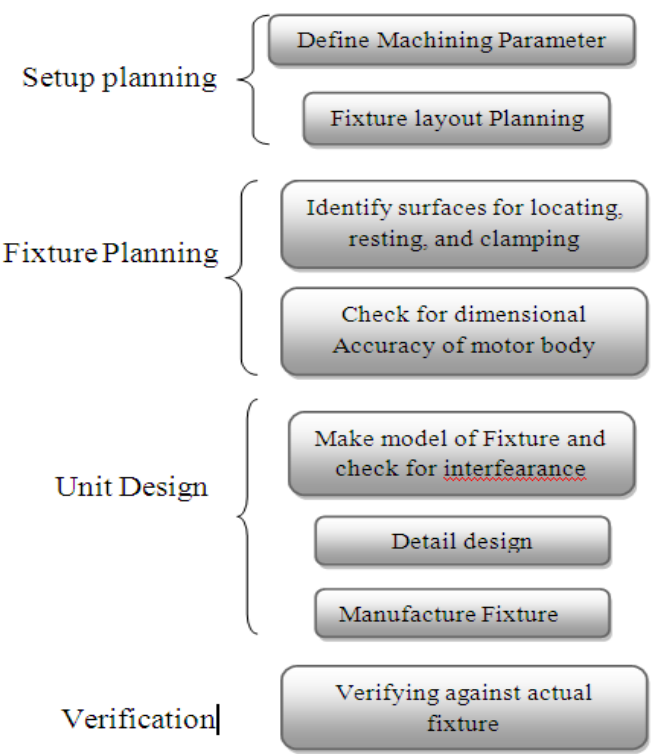

Fig. 4 Fixture Development Process

From the motor body dimension as per given in the Table 1 . We can easily find out the locating, resting and clamping surfaces which will give best support during machining operation.

\section{Locating position}

We are studied many motor stator body drawing for the finding such position for similar location. We have found following locating surfaces in motor body:

- At Spigot ID

- At finished bore

- At bore relief ID 
IOSR Journal of Engineering

Apr. 2012, Vol. 2(4) pp: 768-772
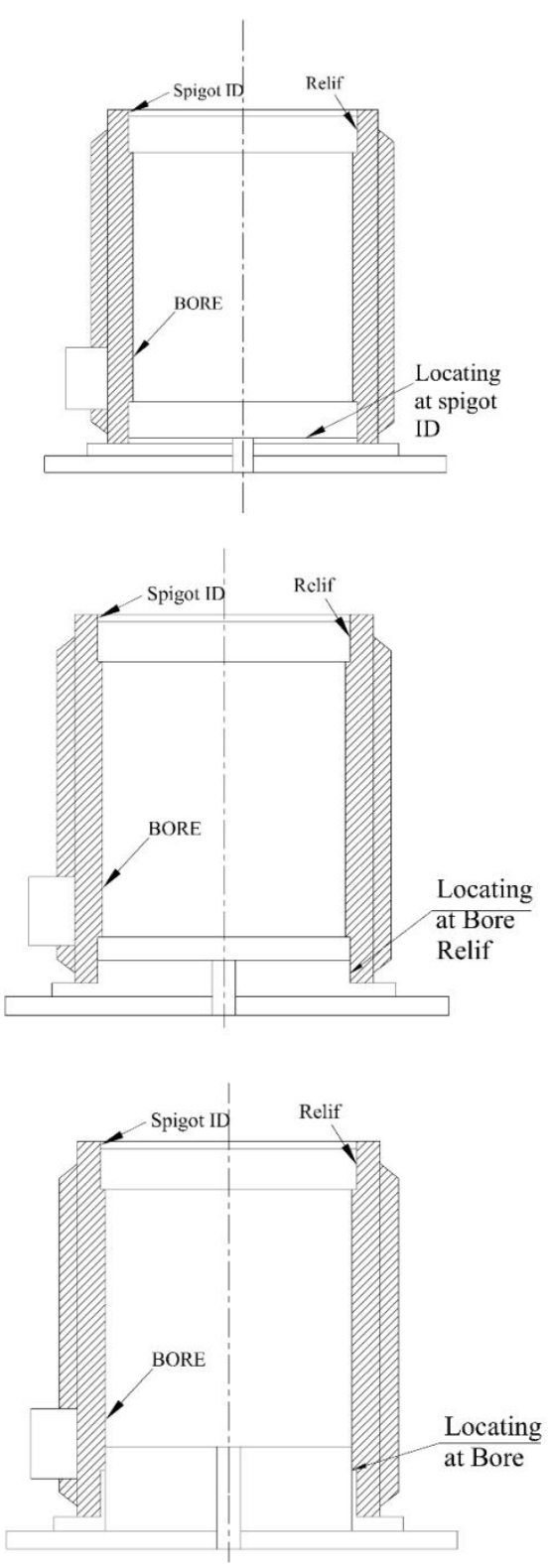

Fig. 5. Possible locating position

These three surfaces we can use for location. Where in a spigot just $10 \mathrm{~mm}$ space available for locating and this spigot has accuracy is within $0.050 \mathrm{~mm}$. While finished bore has accuracy is within a $0.050 \mathrm{um}$ and we can us up to $100 \mathrm{~mm}$ depth for locating. And for the bore relief is accuracy is $0.5 \mathrm{~mm}$ and available depth is around $100 \mathrm{~mm}$.

\section{Resting position}

We can use a resting position as below

- Bore start

- Face of motor body

- Bore Relief start
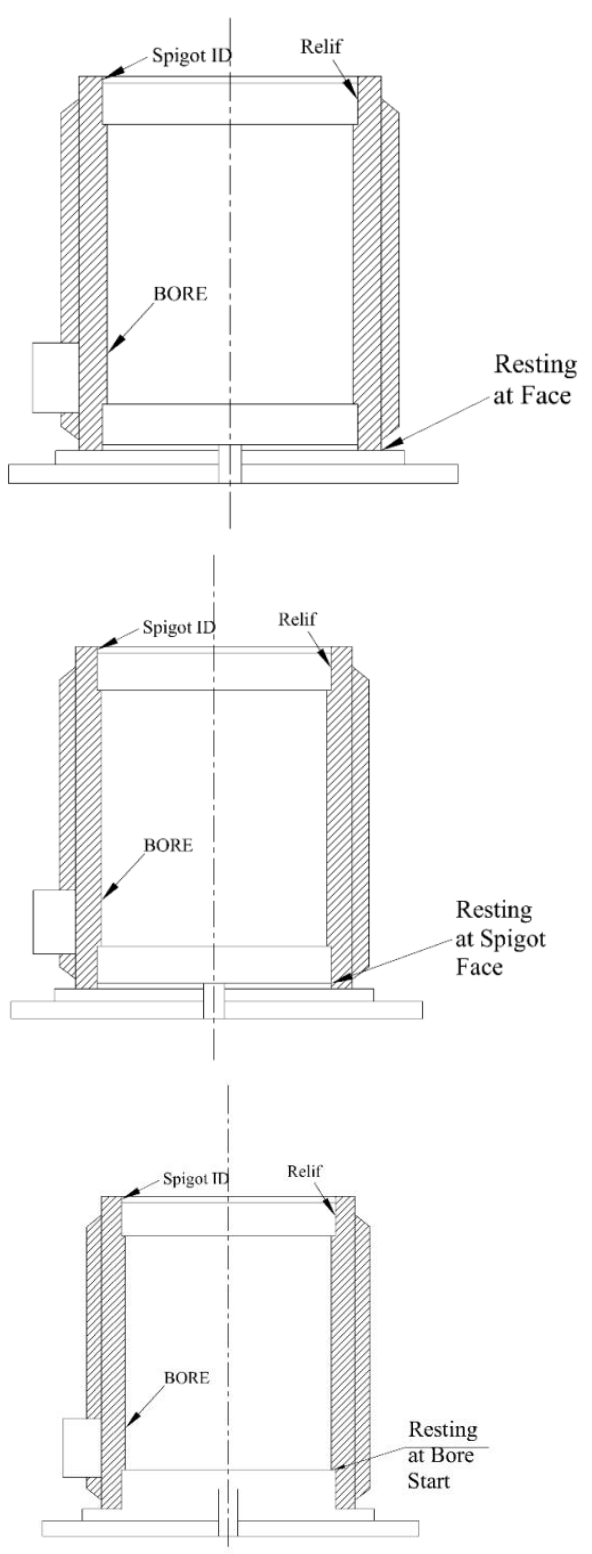

Fig 6. Possible resting position

This three reference can identify as the resting position. A bore start is a point where bore is starting and the thickness of that part is half of diametrically difference between bore and bore relief diameter. This thickness is very low and is about 0.5 to $5 \mathrm{~mm}$ max. There for it isn't provide good support for the workpiece resting. Face of a motor body give good surface for resting position and it is around $50 \mathrm{~mm}$. so a good support is provided by the face during machining operation. A face is finished surface and it's flatness is controlled within $0.050 \mathrm{~mm}$. Due to large surface area in contact with fixture surface, frictional resistant helps to provide stability against cutting force. A bore relief start has very low thickness and it is about half of the diametrically difference between relief is and spigot ID which is around 10 to $15 \mathrm{~mm}$ maximum. Therefore that such surface area is very low to give a support to a whole motor body. 


\section{Clamping position}

As per the 3-2-1 fixture principle clamping position of any component should be perpendicular to the resting position so the clamping force can be directly transferred to the resting pad. This clamping position also reduce deformation of component during clamping position. We can identify much more clamping position for motor stator body as motor body arrives but the clamping posing should not be a position where a cutting tool path is obstructed. This position can be identified by viewing the drawing. But top face is very useful for the clamping. A top surface is machined surface and it is good for the clamp resting. Upper top surface is machined so it can transfer whole lode to the resting pad without deforming the body.

\section{Conclusion}

Motor stator body is very complicated part due to its dimensional accuracy and thin wall thickness. Lots of series available in motor stator body and all are very similar to its feature configuration with dimension changes. So we can easily make fixture which can be used for multiple motor body with changing one or two component in fixture assembly. With help of blackboard approach fixture development process can be accomplish easily and within a short duration.

\section{Referance}

A. Iain Boyle a, YimingRong, David, Brown "A review and analysis of current computer-aided fixture design approaches", Robotics and Computer-Integrated Manufacturing 27 (2011)1-12

B. Utpal Roy, Jianmin Liao, "Application of a blackboard framework to a cooperative fixture design system" , Computers in Industry 37 _1998. 67-81

C. Mhrkus, Zs. Ruttkay and J. Vfincza, "Automating Fixture Design From Imitating Practice to Understanding Principles", Computer and Automation Institute, Hungarian Academy of Sciences, Hungary

D. J.R. Boerma, H.J.J. Kals, "Fixture design with FIXES: the automatic selection of positioning, clamping and support features for prismatic parts", Ann. CIRP 381. 1989. 399-402

E. M. Y. Wang and D. Pelinescu, "Optimizing fixture layout in a point-set domain," IEEE Trans. on Robotics and Automation, vol. 17, no. 3, pp. 312 - 323, 2001

F. Krsulja M.; Barisic B. \& Kudlacek J. “Assembly Setup For Moduer Fixturing Machining process" Advance Engineering, 3(2009)1, ISSN 1846-5900

G. An Advanced Tecnic on Fixture Design and Planning by Andrew Y C Nee, A Senthil Kumar, Z J Tao pp.2147

H. Otto Jan Bakker, "Control Methodology and Modeling of Active Fixtures", The University of Nottingham, August 2010

I. Necmettin Kaya "Machining fixture locating and clamping position optimization using genetic algorithms", Computers in Industry 57 (2006) 112-120

J. Bo Li, Shreyes N. Melkote, "Improved workpiece location accuracy through fixture layout optimization", International Journal of Machine Tools \& Manufacture 39 (1999) 871-883

K. M, Zhang W. J. "Flexible fixture design and automation: review, issues, and future directions .Int. Prod. Res2001;39(13):2867-94. 